OPEN ACCESS

Edited by: Stefania Ceruti, University of Milan, Italy

Reviewed by: Kimberly Nixon, University of Texas at Austin, United States Simon Thomas Schafer. Salk Institute for Biological Studies, United States

${ }^{*}$ Correspondence: Stella E. Tsirka styliani-anna.tsirka@stonybrook.edu

Received: 09 October 2018 Accepted: 27 February 2019 Published: 02 April 2019

Citation: Kyle J, Wu M, Gourzi S and Tsirka SE (2019) Proliferation and Differentiation in the Adult Subventricular Zone are Not Affected by CSF1R Inhibition. Front. Cell. Neurosci. 13:97. doi: 10.3389/fncel.2019.00097

\section{Proliferation and Differentiation in the Adult Subventricular Zone Are Not Affected by CSF1R Inhibition}

\author{
Jackson Kyle, Michelle Wu, Stefania Gourzi and Stella E. Tsirka* \\ Molecular and Cellular Pharmacology Graduate Program, Department of Pharmacological Sciences, Stony Brook University, \\ Stony Brook, NY, United States
}

Microglia are reported to have significant roles in regulating normal mammalian adult neurogenesis. There are two neurogenic niches in the adult mammal brain: the subgranular zone (SGZ) in the hippocampus, and the subventricular zone (SVZ), which makes up the lining of the lateral ventricles. While the microglia interactions on adult neurogenesis in the hippocampus have been characterized, the SVZ niche is not as well investigated. The SVZ niche is unique in that the newborn neurons migrate a much longer distance through multiple brain structures compared to newborn neurons in the hippocampus, making it more difficult to fully characterize how microglia influence this process. To examine the SVZ niche and migration pathway, we used the colony stimulating factor 1 receptor (CSF1R) antagonist PLX5622, which promotes brain wide microglia ablation. Microglia ablation resulted in no changes in the numbers of neural stem cells (NSCs), transient amplifying cells, and neuroblasts. Microglia ablation in the olfactory bulb (OB) was decreased compared to the SVZ. CSF1R inhibition had no effect on the ability of microglia to proliferate. Thus, our data suggest that microglia are not required for normal functioning SVZ adult neurogenesis.

Keywords: CSF1R, microglia, PLX5622, adult neurogenesis, neural stem cells

\section{INTRODUCTION}

In the adult mammalian brain, new neurons are continuously born from neural stem cells (NSCs; reviewed in Bond et al., 2015). These NSCs reside in two niches in the adult brain-the subventricular zone (SVZ), which forms the lining of the lateral ventricles, and the subgranular zone (SGZ) in the hippocampus. In the SVZ, NSCs give rise to transit amplifying cells, which can produce neural progenitors called neuroblasts. Neuroblasts migrate out of the SVZ niche to the olfactory bulb (OB) via the rostral migratory stream (RMS) following "chain migration" (Lois et al., 1996). In the OB neuroblasts mature into neurons, but few become integrated into the existing neuronal circuits. SVZ-OB neurogenesis functions in odor recognition in mammals, while hippocampal neurogenesis is important for memory and learning (Reshef et al., 2014). In pathological conditions and injury, such as ischemic stroke, stimulated NSCs proliferate in both the SVZ and SGZ (Kokaia and Lindvall, 2003; Parent, 2003). Newborn neurons from the SVZ have been reported to divert from their migration along the RMS and instead migrate towards the lesion site. However, most of these neurons do not survive long term (Arvidsson et al., 2002). 
Other cell types populating the neurogenic niche microenvironment have been shown to influence adult neurogenesis, including microglia. Microglia are the resident immune cells of the central nervous system (CNS; reviewed in Li and Barres, 2018). They become activated in response to injury or infection, becoming either classically activated pro-inflammatory (M1-like), or alternatively activated anti-inflammatory (M2like). Activated microglia release pro- and anti-inflammatory cytokines, respectively, and both pathways result in increased phagocytosis of cellular debris and pathogens (Butovsky et al., 2006). Recently many studies focus on investigating microglia before they become activated, while they remain in a "resting" state. Resting microglia continuously sample and monitor their local environment with their ramified processes that are constantly motile (Nimmerjahn et al., 2005). In this state, microglia are involved in developmental and adult neuronal pruning and synapse sculpting via the microglial CX3C receptor-1 (CX3CR1) interacting with the neuronal CX3C ligand-1 (CX3CL1; Paolicelli et al., 2011; Reshef et al., 2017).

Microglia have been shown to be important for adult neurogenesis. In culture experiments, microglial conditioned media promoted migration of neuroblasts (Aarum et al., 2003). Pro-inflammatory environments formed by microglia hindered and reduced neurogenesis (Ekdahl et al., 2003). However, microglia can also promote neurogenesis following alternative anti-inflammatory activation accompanied by the secretion of IL-4 (Butovsky et al., 2006; Shigemoto-Mogami et al., 2014). In the hippocampus, phagocytosis of apoptotic neuroblasts and progenitors by microglia was shown to be important for the proper functioning of local adult neurogenesis (Sierra et al., 2010). In the SVZ, however, it is not as clear. Microglia in the adult rodent SVZ do not express TREM2 (triggering receptor expressed on myeloid cells 2), a receptor involved in phagocytosis (Takahashi et al., 2005), suggesting that regular clearing of cells by microglia does not happen in the SVZ (Sierra et al., 2010; Ribeiro Xavier et al., 2015). However, injections of saporin conjugated to CD11b, a toxin which depletes microglia locally at the SVZ, resulted in decreased numbers of neuroblasts in the RMS and OB and an increase in the SVZ (Ribeiro Xavier et al., 2015). Additionally, genetic ablation of TAM receptor kinases Mer and Axl, which are involved with phagocytosis of apoptotic cells, result in an increase of apoptotic cells in the SVZ and RMS (Scott et al., 2001; Fourgeaud et al., 2016). Thus, it remains unclear how exactly microglia influence the SVZ, RMS, and $\mathrm{OB}$ niche.

Microglial roles have been investigated extensively through the use of reagents that result in their ablation. Such reagents include clodronate delivered in liposomes, which has been shown to yield a $70 \%-80 \%$ efficiency in microglial depletion (Torres et al., 2016; Nelson and Lenz, 2017), and Mac-1Saporin which results in about 50\% depletion of the cells (Yao et al., 2016; Han et al., 2017). Other compounds target the colony stimulating factor-1 receptor (CSF1R) expressed on microglia. CSF1R signaling is required for microglia survival, and blocking of the receptor with an antagonist results in brain wide ablation (Elmore et al., 2014). One of the compounds used is GW2580, which has been reported to inhibit CSF1R both in microglia and macrophages: when the compound was used in a model of renal and neuropsychiatric lupus lower ionized calcium binding adaptor molecule 1 (Iba1+, a marker for microglia and macrophages) counts were reported in the kidney glomeruli (Chalmers et al., 2017), but did not yield any significant change in the total number of microglial cells in normal conditions (Olmos-Alonso et al., 2016). In a model of spinal cord injury, GW2580 resulted in a 60\% reduction of microglia in the lesion epicenter (Gerber et al., 2018). Another CSF1R inhibitor, BLZ945, was efficient in depleting microglia in a dose-dependent manner in the uninjured CNS, but was less effective in depleting activated microglia (Beckmann et al., 2018). A series of CSF1R inhibitors have been developed by Plexxikon Inc., which have been shown to be capable to deplete microglia. The first of these compounds was PLX3397, which in addition inhibits c-Kit (Elmore et al., 2014), and has been reported to achieve $90 \%-99 \%$ depletion of microglia (Szalay et al., 2016; Jin et al., 2017; Groh et al., 2019). In this study, we use a different microglial inhibitor, PLX5622, which does not act on c-Kit, and has been reported to yield $\sim 90 \%$ microglial ablation (Acharya et al., 2016; Walter and Crews, 2017; Nissen et al., 2018; Seitz et al., 2018; Unger et al., 2018). It is delivered orally in the mouse chow, and this non-invasive delivery methods is preferred to local injections or infusion of other compounds that result in (minimal) mechanical injury in the tissue and accompanying microglial activation (Torres et al., 2016). Of clinical significance, the ablation is reversible, with almost complete repopulation of microglia in the brain by day 3 after withdrawal of PLX5622 treatment (Dagher et al., 2015). Recently PLX5622 was used to study adult neurogenesis in the $\mathrm{OB}$, but not in the other SVZ-OB pathway regions. In the OB, changes in neuronal spine density and spine dynamics were seen. However, no changes were seen in neuroblast or mature neuron populations (Reshef et al., 2017). We study in this report microglia/neurogenesis interactions in all regions of SVZ-OB of adult mice. We find that microglia are not required for normal proliferation and differentiation in the adult SVZ and RMS, and we report that CSF1R inhibition via PLX5622 does not affect microglia proliferation rates.

\section{MATERIALS AND METHODS}

\section{Animals}

Experiments were performed on 9-week-old male C57BL/6 mice bred in maximum isolation with $12 / 12 \mathrm{~h}$ light/dark cycle conditions. Animals had free access to food and water, and were kept on standard mice chow until experiments were started. Mice were group housed before and during experiments. All experiments were performed using protocols approved by the Stony Brook University Institutional Animal Care and Use Committee (IACUC).

\section{Microglia Ablation}

CSF1R antagonist PLX5622 (1,200 ppm; 1,200 mg/kg) was provided by Plexxikon, and is formulated in AIN-76A chow by 
Research Diets. Mice were fed PLX5622 or control chow (AIN$76 \mathrm{~A}$ ) for 7 or 14 days, resulting in brain wide microglia ablation (Dagher et al., 2015).

\section{BrdU Administration}

5-bromo-2'-deoxyuridine (BrdU, Sigma B5002) was administered in drinking water $(1.0 \mathrm{mg} / \mathrm{ml})$ supplemented with $1 \%$ sucrose to encourage drinking. Twenty five millilitters of BrdU water per mouse were given during the last 4 days of PLX5622 treatment. While the amount of water consumed was not measured, we ascertained daily that the mice had enough water. At the end of the experimental period a small volume if any of water was left. Additionally, Ki67 staining was performed on sections to further validate the BrdU results.

\section{Immunohistochemistry}

At the 7-day (7d) or 14-day (14d) time point, mice were deeply anesthetized with $2.5 \%$ Avertin, were transcardially perfused with $25 \mathrm{mLs} 1 \times$ phosphate buffered saline (PBS) followed by $25 \mathrm{mLs}$ $4 \%$ paraformaldehyde (PFA) in $1 \times$ PBS. Brains were collected and post-fixed in 4\% PFA overnight for two nights and then placed in $1 \times$ PBS until sectioned. Brains were mounted, covered in $1 \times$ PBS, and sagittal sections were collected with a Leica Vibratome VT 1000S. Tissue within $2 \mathrm{~mm}$ laterally of the midline were sectioned into $50 \mu \mathrm{m}$ slices and serially collected in a 12 well plate containing $1 \times \mathrm{PBS}$. Plates were stored in $4^{\circ} \mathrm{C}$ until wells of tissue were used.

Free floating sections were incubated for $2 \mathrm{~h}$ in blocking solution containing 10\% normal donkey (Sigma D9663) or goat serum (Southern Biotech 100241) and 0.3\% Triton-x100 in $1 \times$ PBS. Immediately after blocking, tissue was incubated with primary antibodies overnight at $4{ }^{\circ} \mathrm{C}$ in dilution solution ( $1 \%$ bovine serum albumin, $0.3 \%$ Triton-x100 in $1 \times$ PBS; Table 1). After PBS washes, sections were incubated with secondary antibodies in dilution solution for $1 \mathrm{~h}$ at room temp in the dark. Following another round of PBS washes, slices were mounted on slides (VWR Micro Superfrost Plus) and cover slipped (VWR Micro Cover Glass) with DAPI Fluoromount (SoutherBiotech G0100). The slides were left in the dark at room temperature overnight, and then sealed with

\begin{tabular}{llll}
\hline \multicolumn{2}{l}{ TABLE 1 | Antibodies used in experiments. } & \\
\hline Antigen & Species & Dilution & Supplier \\
\hline Iba1 & Rabbit & $1: 500$ & Wako 019-19741 \\
Sox2 & Mouse & $1: 200$ & R\&D Systems MAB2018 \\
GFAP & Rabbit & $1: 500$ & Dako Z0334 \\
Ki67 & Rat & $1: 500$ & Fisher 245-564 \\
DCX & Goat & $1: 200$ & Santa Cruz sc-8066 \\
BrdU & Rat & $1: 500$ & Novus NB500-1690 \\
NG2 & Rabbit & $1: 500$ & Dr. Joel Levine, SBU \\
CC1 & Mouse & $1: 100$ & CalBioChem OP80 \\
CSF1R & Rabbit & $1: 200$ & Santa Cruz sc-692 \\
Cleaved caspase 3 & Rabbit & $1: 200$ & Cell Signaling D175 \\
Alexa donkey anti-rat 488 & Donkey & $1: 1,000$ & Life Technologies A21208 \\
Alexa donkey anti-rabbit 647 & Donkey & $1: 1,000$ & BioLegend 406414 \\
Alexa goat anti-rabbit 488 & Goat & $1: 1,000$ & Life Technologies A11008 \\
Alexa goat anti-mouse 555 & Goat & $1: 1,000$ & Life Technologies A21424 \\
Alexa goat anti-rabbit 633 & Goat & $1: 1,000$ & Life Technologies A21071 \\
Goat anti-rat Cy5 & Goat & $1: 1,000$ & Abcam ab6565
\end{tabular}

clear nail polish. For BrdU staining, tissue was treated with $2 \mathrm{~N}$ hydrochloric acid (HCL) for $1 \mathrm{~h}$, washed with $0.1 \mathrm{M}$ sodium borate, then washed with $1 \times$ PBS before treated with blocking solution.

\section{Imaging and Analysis}

A Leica SP8X confocal microscope was used for imaging. For the SVZ, tilescans (multiple consecutive images that are stitched together to make a single rectangular image) of the whole lateral wall of the SVZ on two slices per brain was imaged at $40 \mathrm{x}$ magnification, consistent with previous publications (Ribeiro Xavier et al., 2015). For the RMS and OB, either three $40 \times$ magnification images for the Ki67/Ibal staining, or five $63 \times$ magnification images for the BrdU/Ibal staining were chosen at random on slices containing the RMS and granular cell layer in the $\mathrm{OB}$ and marked by the confocal software. $100 \times$ magnification was used to analyze single cells. All images were obtained as $10 \mu \mathrm{m}$ z-stacks, with $1 \mu \mathrm{m}$ used for the $\mathrm{z}$-step between each image of the z-stack. Image analysis, including cell counting, was performed on either Leica LAX-S or ImageJ (NIH) software.

\section{Statistical Analysis}

Six mice were used per group (24 mice total), and two sections for each marker were quantified. Two-way analyses of variance (ANOVA) with Bonferroni posttest was used to evaluate data comparing the interaction between region and treatment; Student's $t$-test was used to compare data between treatments within a single region. A $p$-value of $<0.05$ was used to determine significant difference with a $95 \%$ confidence interval. All graphs are plotted as averages \pm SEM.

\section{RESULTS}

\section{CSF1R Inhibitor, PLX5622, Treatment Has Negligible Effect on Adult Neurogenesis}

To inquire whether the PLX5622 diet effectively ablated microglia in our system, immunofluorescence for Iba1, a marker for microglia/macrophages, was performed (Figures 1A,B, Supplementary Figure S1A). At 14 days (14d) of treatment, there were higher numbers of microglia in the $\mathrm{OB}$ compared to the SVZ in both control and PLX5622 treated mice, and in the RMS compared to the SVZ in control mice (Figure 1C). After 7 days $(7 \mathrm{~d})$, microglial quantification showed significant levels of ablation compared to the respective controls in the SVZ, RMS, and OB (Supplementary Figure S1B). On average, ablation levels varied from $74 \%$ to $93 \%$. However, the OB displayed lower levels of ablation overall compared to the SVZ after either the $7 \mathrm{~d}$ and $14 \mathrm{~d}$, and in the RMS compared to the SVZ at $7 \mathrm{~d}$ but not 14d (Figure 1D). Overall, PLX5622 treatment for $7 \mathrm{~d}$ or $14 \mathrm{~d}$ resulted in brain-wide microglia ablation in the SVZ, RMS, and OB. Since ablation percentages were slightly higher after $14 \mathrm{~d}$ of PLX5622 treatment than $7 \mathrm{~d}$ of treatment, $14 \mathrm{~d}$ data are discussed more extensively in the subsequent experiments.

Changes in neuroblast migration were evident after microglial ablation in the SVZ via saporin conjugated to CD11b (Ribeiro Xavier et al., 2015). To determine if PLX5622 treatment 


\section{A}
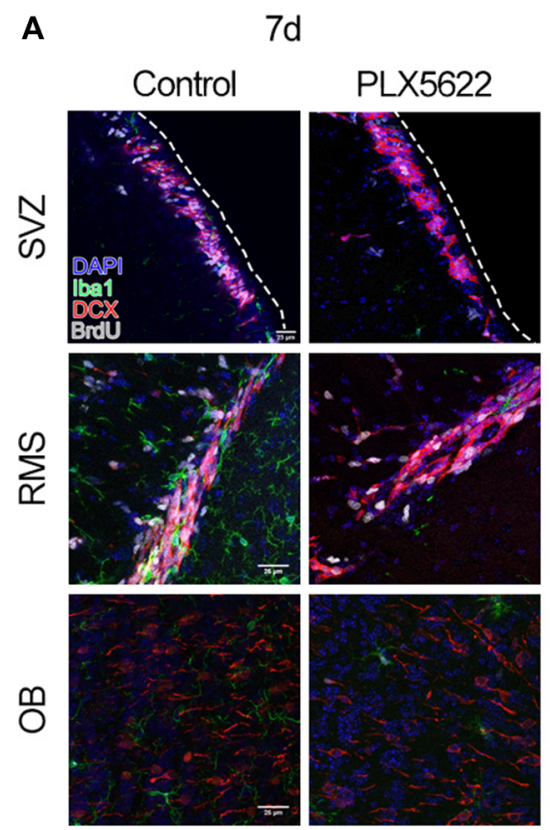

C

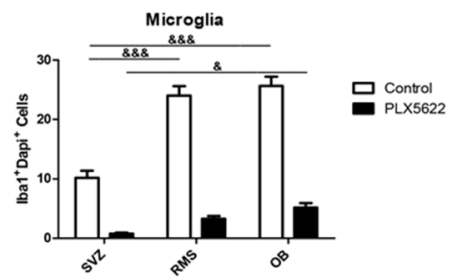

E

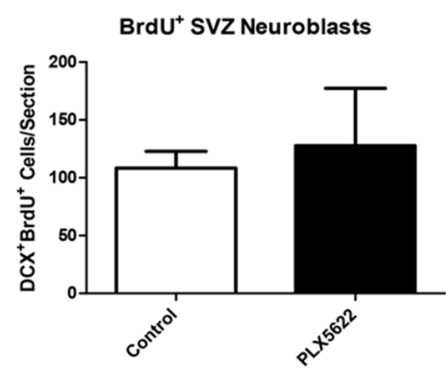

G

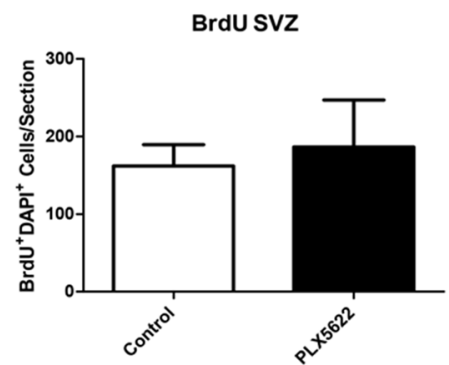

PLX5622
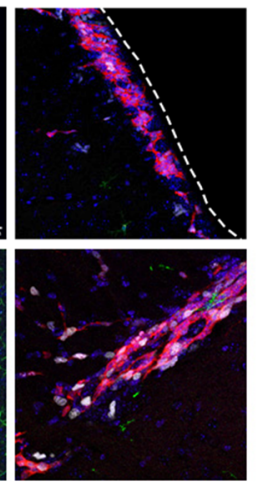

B
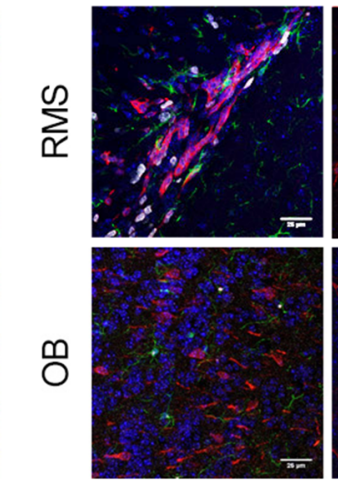

D Microglia Ablation

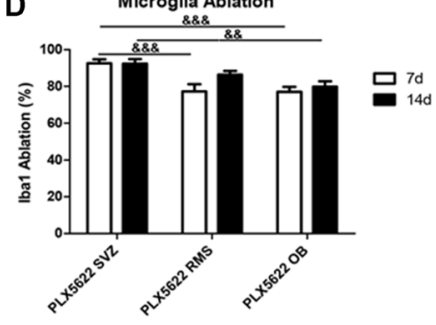

$F$

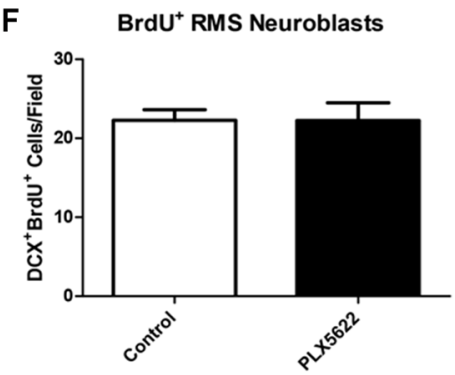

H

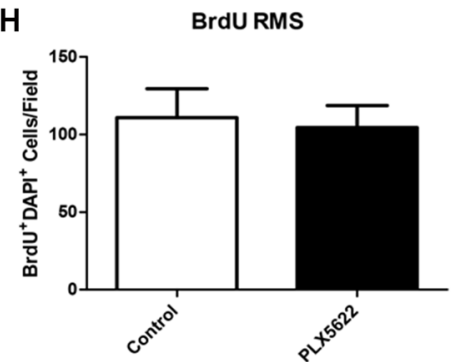

FIGURE 1 | Brain wide microglia ablation results in no change on neuroblast production. Representative images of the subventricular zone (SVZ), rostral migratory stream (RMS), and olfactory bulb (OB) from 7 days (7d; A) and 14 days (14d; B) of PLX5622 treatment, with the dashed line separating the SVZ from the lateral ventricle. DAPI in blue, ionized calcium binding adaptor molecule 1 (Iba1) in green, doublecortin (DCX) in red, and 5-bromo-20-deoxyuridine (BrdU) in gray. Quantification of 14d microglia ablation shown in $\left(\mathbf{C}, F_{(2,15)}=13.41, p=0.0005\right)$. The ablation percentage was calculated by dividing the PLX5622 Iba1+ cell count for a brain from the average of the control, respective to each region $\left(\mathbf{D}, F_{(2,15)}=1.56, p=0.22\right)$. Quantification of $14 \mathrm{~d}$ BrdU positive neuroblasts shown in $(\mathbf{E}$, $p=0.7091)$ and $(\mathbf{F}, p=0.9902)$, and $14 d$ total BrdU in $(\mathbf{G}, p=0.7168 ; \mathbf{H}, p=0.7863) .{ }^{*}=$ comparison between regions within treatment. \& $=p<0.5$;

$\& \&=p<0.01 ;{ }^{\& \& \&}=p<0.001 . n=6$ for all groups, two sections per $n$. Microglia in (C) plotted as an average per section generated by sum of field of views. 

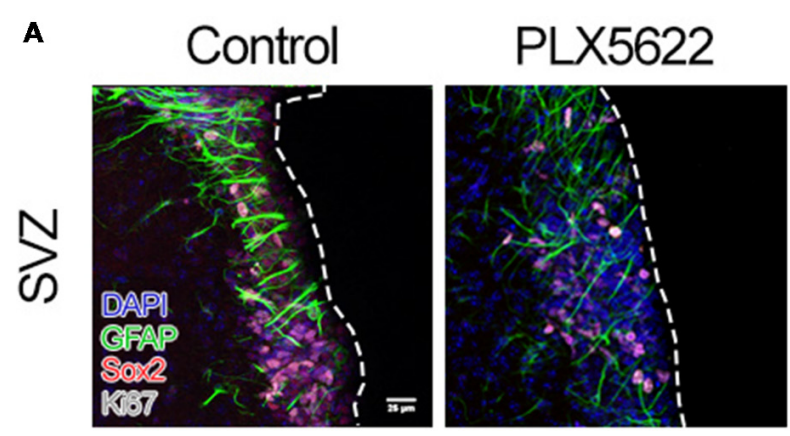

\section{B}

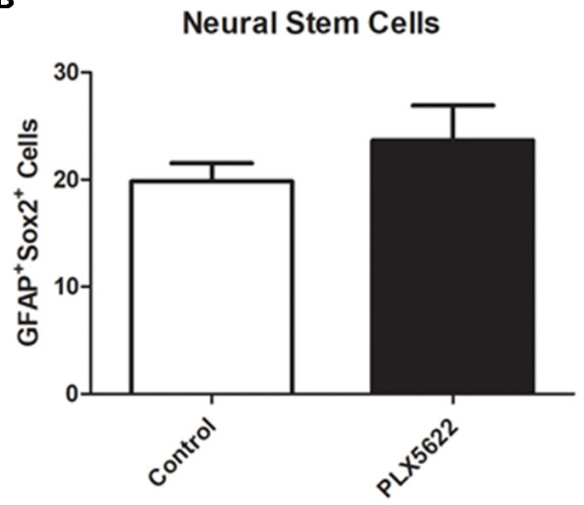

C

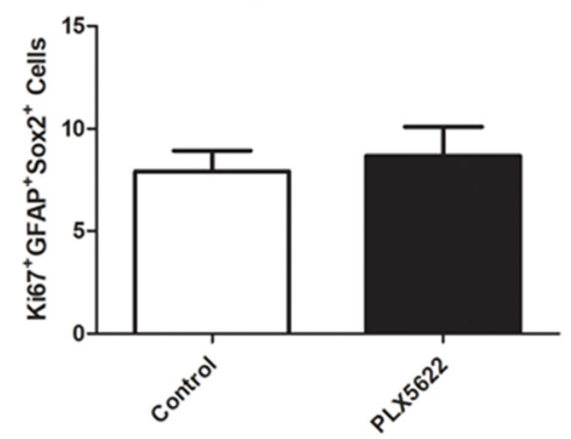

$\mathbf{D}$

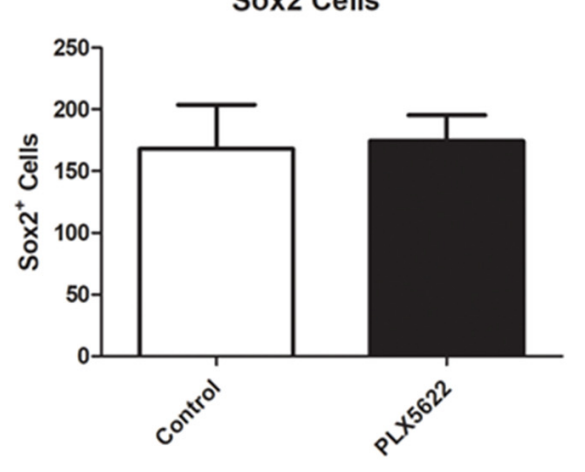

FIGURE 2 | No change in neural stem cell (NSC) or TAC population from PLX5622 treatment. Representative images of the SVZ from 14d of PLX5622 treatment (A), with the dashed line separating the SVZ from the lateral ventricle. DAPI in blue, glial fibrillary acidic protein (GFAP) in green, Sox2 in red, and Ki67 in gray. Quantification of NSC population shown in (B, $p=0.2465)$, with proliferating (Ki67+) NSCs shown in (C, $p=0.6871)$. Sox2+ cells, which includes NSCs and TACs, is shown in (D, $p=0.8964) . n=6$ for all groups, two sections per $n$, plotted as per SVZ wall.

resulted in similar changes, the newly formed and migrating neuroblasts were labeled, through co-immunostaining with doublecortin (DCX), a microtubule protein expressed in neuroblasts, and BrdU to mark neuroblasts that had been produced since its administration (Figures 1A,B). BrdU was provided to the mice in the water 4 days before the end of either the 7d or 14 time point. Mice treated with PLX5622 for 7d had less BrdU ${ }^{+}$neuroblasts in the SVZ (Supplementary Figure S1C). Surprisingly, this difference no longer existed after $14 \mathrm{~d}$ of PLX5622 treatment, and there were no differences in the RMS at either timepoint (Figures 1E,F, Supplementary Figure S1D). To account for labeling cell populations other than just neuroblasts, the numbers of all $\mathrm{BrdU}^{+}$cells were counted in the SVZ and RMS (Figures 1G,H, Supplementary Figures S1E,F). Corresponding with the neuroblast counts, there was a decrease in the SVZ at 7d of PLX5622 treatment, that was no longer observed by $14 \mathrm{~d}$. There was no significant difference between control and PLX5622 treated mice within the RMS at either timepoint. Since the 4 days of BrdU administration may not be enough time for most BrdU labeled neuroblasts to migrate to the OB (Petreanu and Alvarez-Buylla, 2002), the analysis performed did not include the OB.

To determine if microglia ablation from PLX5622 treatment influenced the NSCs in the SVZ, staining with glial fibrillary acidic protein (GFAP) and SRY box 2 (Sox2) was performed. Additionally, Ki67, a marker for cellular proliferation, was used to label NSCs that were actively proliferating (Figure 2A, Supplementary Figure S2A). Upto 14d of PLX5622 treatment, there was no change in the numbers of NSCs in the SVZ (Figure 2B, Supplementary Figure S2B); nor was there any change in the numbers of proliferating NSCs following PLX5622 treatment (Figure 2C, Supplementary Figure S2C). As TACs express Sox2, total SVZ Sox2 was quantified; no changes in the numbers of $\mathrm{Sox} 2^{+}$cells in the SVZ after PLX5622 treatment were evident (Figure 2D, Supplementary Figure S2D).

NSCs in the adult SVZ may also differentiate into oligodendrocytes (oligos), but in lower numbers than TACs/neuroblasts. This differentiation is typically observed on the superior wall of the SVZ, but not in the lateral wall (Menn et al., 2006; Ortega et al., 2013). To examine if microglia influence adult oligogenesis in the SVZ, a marker for mature oligos [anti-adenomatous polyposis coli clone CC1 (CC1)] and neural/glial antigen 2 (NG2), a marker for oligo progenitors, were used to image the superior wall of the SVZ (Figure 3A, Supplementary Figure S3A). Again, there were no differences after 14 or $7 \mathrm{~d}$ of PLX5622 treatment compared to control in either numbers 


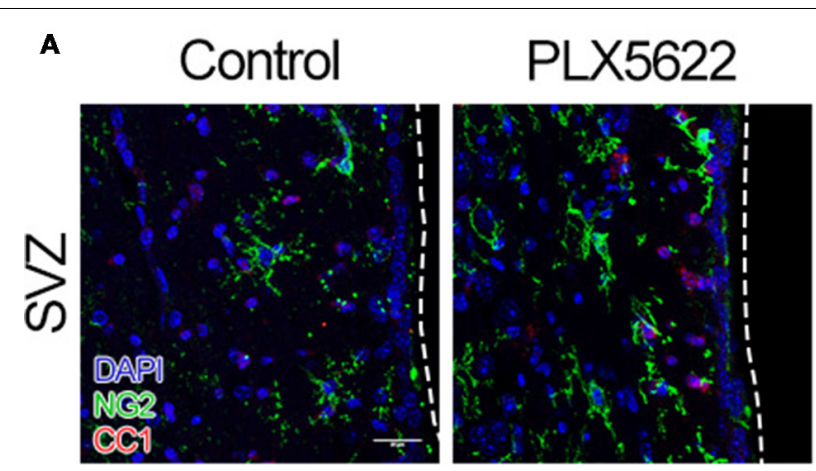

B

Mature Oligos

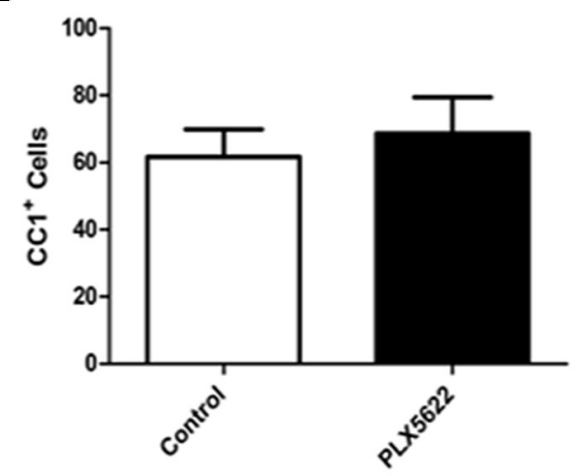

C

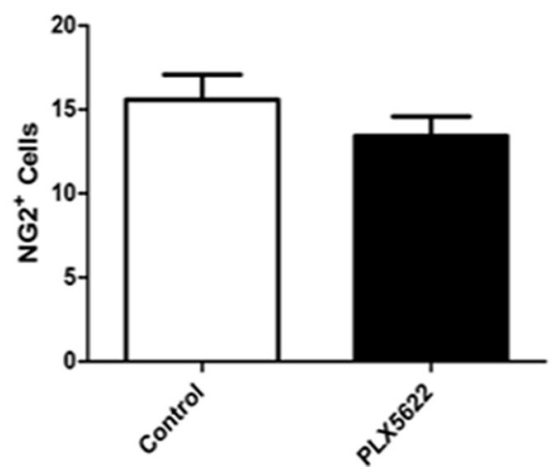

FIGURE 3 | No change in oligo precursors and mature oligos from PLX5622 treatment. Representative images of the SVZ from 14d of PLX5622 treatment (A), with the dashed line separating the SVZ from the lateral ventricle. DAPI in blue, neural/glial antigen 2 (NG2) in green, and CC1 in red. Quantification of mature oligos in $(\mathbf{B}, p=0.6101)$ and oligo precursors in $(\mathbf{C}, p=0.2670) . n=6$ for all groups, two sections per $n$ plotted as per SVZ wall.

of mature oligos or oligo precursor cells (Figures 3B,C, Supplementary Figures S3B,C).

As mentioned earlier, TAM receptor ablation has resulted in increased numbers of apoptotic cells. We therefore stained for cleaved caspase 3 (CC3) to investigate levels of apoptosis in the SVZ, RMS, and the dentate gyrus (DG) of the hippocampus, where apoptotic cells are reported to be cleared by microglia (Sierra et al., 2010). Surprisingly, there was no increase in apoptotic cells in any of these regions after PLX5622 treatment (Supplementary Figure S4). Taken together, these data suggest that microglia may not be required for normal adult neurogenesis in the SVZ niche.

\section{CSF1R Inhibition via PLX5622 Has No Effect on Microglia Proliferation Rates}

To understand better whether CSF1R inhibition by PLX5622 affected resting or active/proliferating microglia in the SVZ niche, cells were co-labeled with BrdU and Iba1 (Figures 1A,B). The total number of microglia counted for control SVZ, RMS, and OB were 122, 288, and 308, respectively. For PLX5622 SVZ, RMS, and OB, 9, 39, and 62 cells were counted. BrdU ${ }^{+}$microglia were quantified as having punctate BrdU labeling in the microglia somata, as reported by others (Tay et al., 2017). Interestingly, the control OB had more microglia labeled with BrdU than the SVZ and RMS (Figure 4A, Supplementary Figure S5A; the number of microglia that were $\mathrm{BrdU}^{+}$in control SVZ, RMS, and OB were 24, 81, and 184, whereas for PLX5622 SVZ, RMS, and OB, there were 9, 23, and 34 double positive cells). Despite being so few microglia remaining after PLX5622 treatment, all treated regions had microglia that expressed BrdU, including the $\mathrm{OB}$ which contained significantly more residual microglia than the SVZ. To normalize the different regions, the percentage of $\mathrm{BrdU}^{+}$ microglia was generated by dividing $\mathrm{BrdU}^{+}$microglia by the total number of microglia in the region for each brain; the numbers were then averaged (Figure 4B, Supplementary Figure S5B). Surprisingly, PLX5622 treated mice had no significant change in the percentage of $\mathrm{BrdU}^{+}$microglia from control mice in the $\mathrm{OB}$, but there was a significant increase in the RMS compared to control. Additionally, the OB had an almost identical percentage of $\mathrm{BrdU}^{+}$microglia in both control and PLX5622 treated mice. The PLX5622 SVZ contained more $\mathrm{BrdU}^{+}$microglia than control animals, but the difference was not statistically significant (since the very low numbers of residual microglia in the SVZ after PLX5622 treatment was not evenly distribute amongst the six animals counted, which rendered the statistics not reliable).

To validate this result, we also quantified Ki67-labeled microglia in the SVZ, RMS, and OB (Figure 5A, Supplementary Figure S6A). Total microglia counted for control SVZ, RMS, and OB were 109, 199, and 375, respectively. For PLX5622 SVZ, RMS, and OB, 3, 35, and 90 cells were counted. Iba1+ Ki67+ microglia were again counted for each region (Figure 5B, Supplementary Figure S6B). As with the BrdU, microglia co-labeled with punctate Ki67 were detected (the number of microglia that were $\mathrm{Ki}^{+} 7^{+}$in control SVZ, RMS, and OB were 23, 89, and 253, whereas for PLX5622 SVZ, RMS, and OB, there were 1,19 , and 61 double positive cells). Upon quantification, similar results were found, with the OB having the most $\mathrm{Ki}_{6}{ }^{+}$ microglia, followed by the RMS (Figure 5C, Supplementary Figure S6C). As with BrdU labeling, PLX5622 treated OB had an almost identical percentage of $\mathrm{Ki}^{+}$microglia as control (Figure 5D, Supplementary Figure S6D). There were no significant differences between control and PLX5622 treated mice at 14d, but there was an increase in PLX5622 treated mice at $7 \mathrm{~d}$ in the RMS. The discrepancies in numbers of Iba1+ BrdU+ and Iba1+ Ki67+ in the RMS data could be attributed to very 


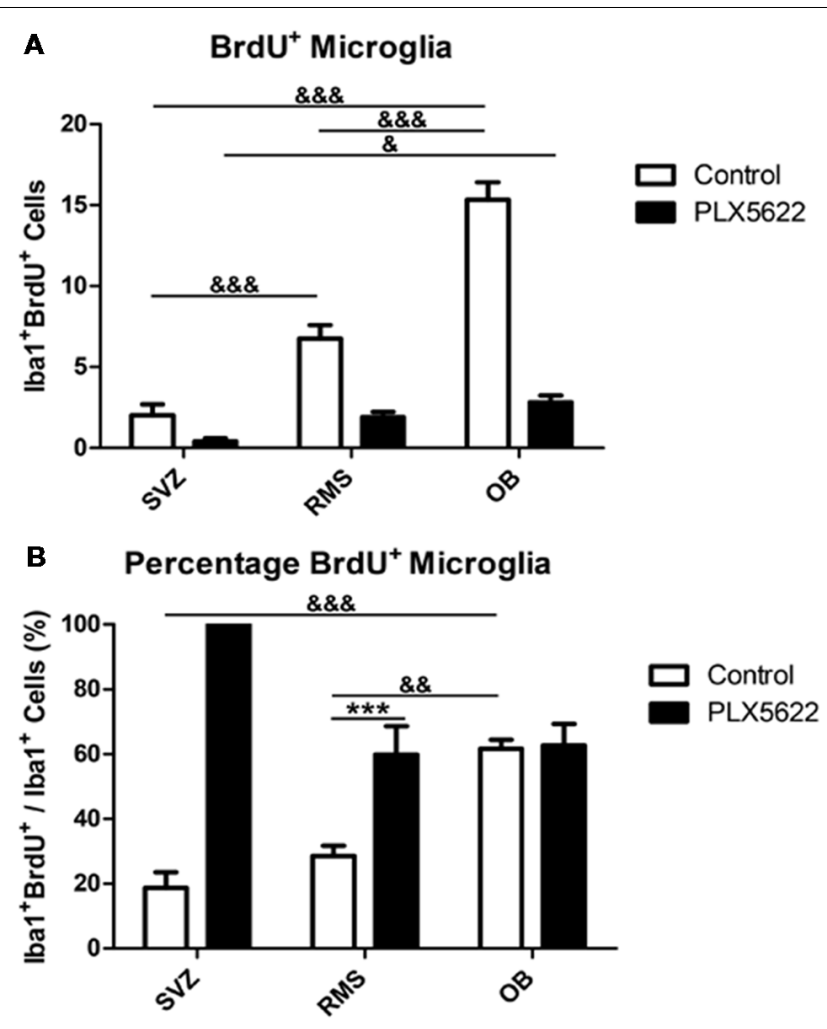

FIGURE 4 | Remaining microglia have no change in rate of replication after PLX5622 treatment. BrdU ${ }^{+} \mathrm{lba1}{ }^{+}$cells were quantified

$\left(\mathbf{A}, F_{(2,15)}=30.39, p<0.0001\right)$ and normalized in $\left(\mathbf{B}, F_{(2,15)}=51.75\right.$, $p<0.0001)$. The $\mathrm{OB}$ contained more $\mathrm{BrdU}^{+}$microglia than the RMS and SVZ in control mice, but only more than the SVZ in

PLX5622 treated mice. When normalized, control OBs had a higher percentage of $\mathrm{BrdU}^{+}$microglia compared to the RMS and SVZ, with no significant difference between control and PLX5622 treated mice.

$\&$ = comparison between regions within treatment, ${ }^{*}=$ comparison between treatments within a region. ${ }^{* / \&}=p<0.5 ;{ }^{* *} / \& \&=p<0.01$; ${ }^{* * *} / \& \& \&=p<0.001 . n=6$ for all groups, two sections per $n$. Microglia in (A) plotted as an average per section generated by sum of field of views.

low overall number of remaining microglia (Iba1+) positive cells from after PLX5622 treatment. Total Ki67 ${ }^{+}$cells were counted in both the SVZ and RMS (Figure 5E, Supplementary Figure S6E). As with BrdU, very few $\mathrm{Ki}^{+} 7^{+}$cells were found in the $\mathrm{OB}$ and almost all that were found were microglia (Figure $\mathbf{5 A}$, Supplementary Figure S6A). There were fewer proliferating cells after PLX5622 treatment, but similar to the total $\mathrm{BrdU}^{+}$cells, these changes were not significant.

To confirm that the microglia in the $\mathrm{OB}$ are sensitive to and would be targeted by the CSF1R inhibitor, CSF1R staining was performed (Supplementary Figures S7A,B). Both the control and PLX5622-treated brains presented with CSF1R expressing cells. $100 \times$ magnification images were taken of CSF1R ${ }^{+}$cells, which had the same shape and structure as ramified microglia. Ki67 labeling was done, and the punctate staining pattern that was seen from BrdU and Ki67 staining with Ibal was observed as before (Sobecki et al., 2016; Tay et al., 2017). The $100 \times$ magnification also helped to ascertain that the Ki67 signal was localized in the cell soma. Taken together, these data suggest that although CSF1R stimulation/signaling is needed for microglia survival, it is not needed for microglia proliferation and replication.

\section{DISCUSSION}

While microglia may influence SVZ adult neurogenesis, the results of the present study suggest that microglia are not required for normal proliferation and differentiation of this process in vivo. We discovered that NSCs populations are not affected by up to $14 \mathrm{~d}$ of PLX5622 treatment (Figures 2B,C). Additionally by $14 \mathrm{~d}$ of treatment, there was no change in neuroblast production (Figure 1E), nor were there changes in oligogenesis (Figure 3). To our knowledge, this is the first study to look at SVZ niche adult neurogenesis after in vivo brain-wide microglia ablation.

In the adult hippocampus, microglia are reported as required to assist in normal, physiological neurogenesis (Sierra et al., 2010). Using a different method of ablation, other labs have locally ablated microglia around the SVZ niche and reported aberrant neuroblast production/migration (Ribeiro Xavier et al., 2015). While we did not explore the hippocampus, our results do not suggest that microglia are needed for normal neuroblast production, migration out of the SVZ, or migration through the RMS. One possible explanation for this is the presence of the supportive "tube" of astrocytes that surround the RMS (Lois et al., 1996) that may be able to substitute for the loss of microglia. Treatment with PLX3397, another compound containing a CSF1R inhibitor, for 7 days resulted in an increase of GFAP mRNA levels, although no difference in astrocyte cell counts were evident (Elmore et al., 2014). Another possible explanation is that, unlike in the hippocampus, phagocytosis of neuroblasts is not a required step for normal SVZ-OB adult neurogenesis. This is supported by the finding that there was no change in the total number of $\mathrm{BrdU}^{+}$cells or $\mathrm{Ki}^{+} 7^{+}$cells in $\mathrm{SVZ}$ and RMS up to 14d of PLX5622 treatment (Figures 1F, 5E). Lastly, the reported change in neuroblast production/migration from saporin-CD11b focal microglia ablation could be due to the release of pro-inflammatory cytokines around the SVZ (Ribeiro Xavier et al., 2015). PLX3397 and PLX5622 treatments have been shown to not result in changes in microglia related cytokine release, such as TNF- $\alpha$ and IL-1 $\beta$ (Elmore et al., 2014; Reshef et al., 2017), thus accounting for a possible difference between these two experimental setups.

One of the main functions of microglia at homeostasis is the phagocytosis and management of neuronal synapses in the OB (Wake et al., 2013). A recent study using a genetic model to specifically ablate Mer and Axl on microglia, which are important receptors for microglial phagocytosis, reported an increased number of BrdU+ cells in the $\mathrm{OB}$ and a higher cell density in the granule and glomerular cell layers. Many of these cells were NeuN+ positive, but others were not (Fourgeaud et al., 2016). In contrast, using PLX5622 to ablate microglia in the $\mathrm{OB}$, no increase in BrdU positive cells in the $\mathrm{OB}$ was seen. Instead, changes in dendritic spines dynamics and lower 


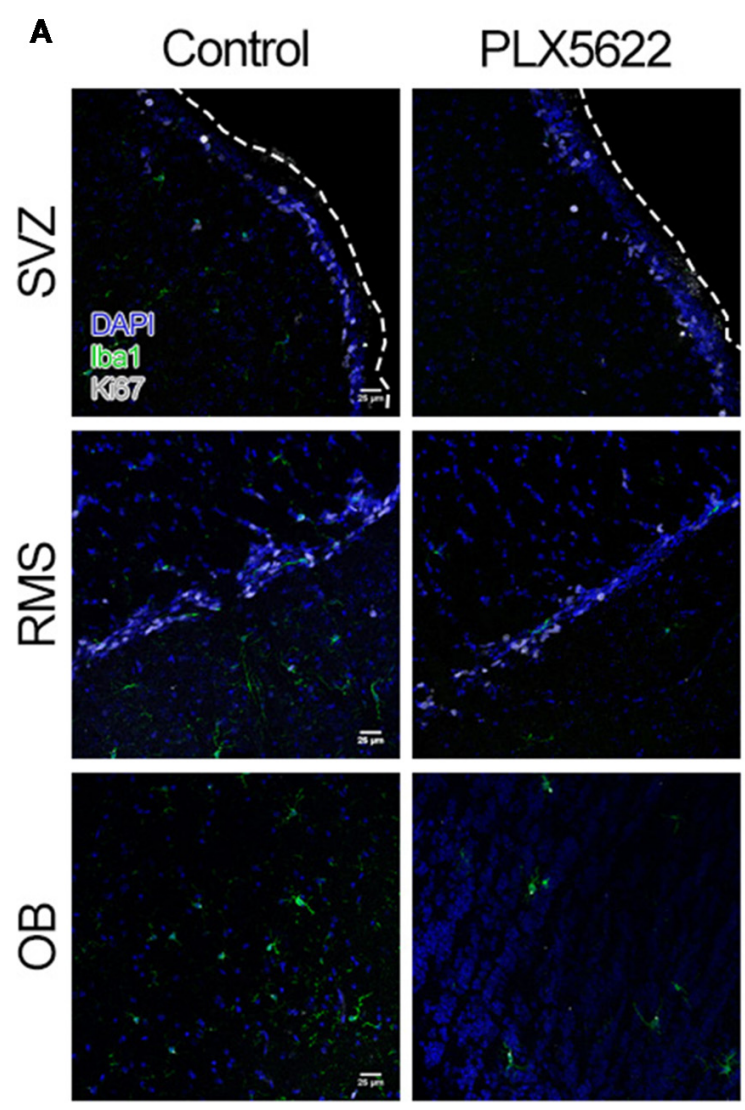

B

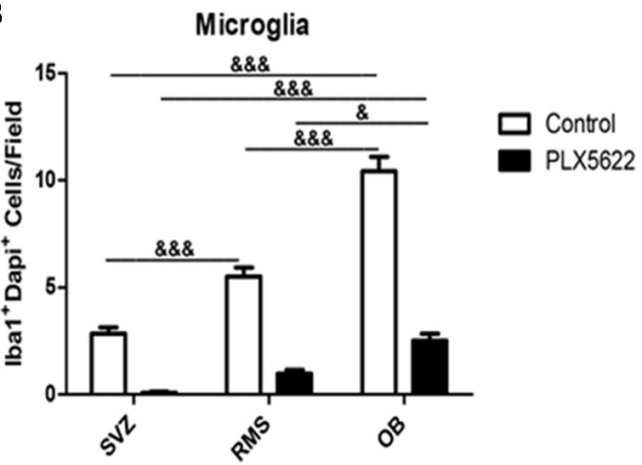

C

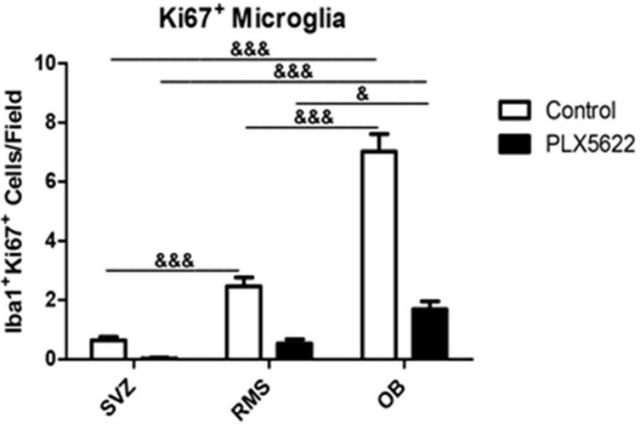

D

Percentage Ki67 ${ }^{+}$Microglia

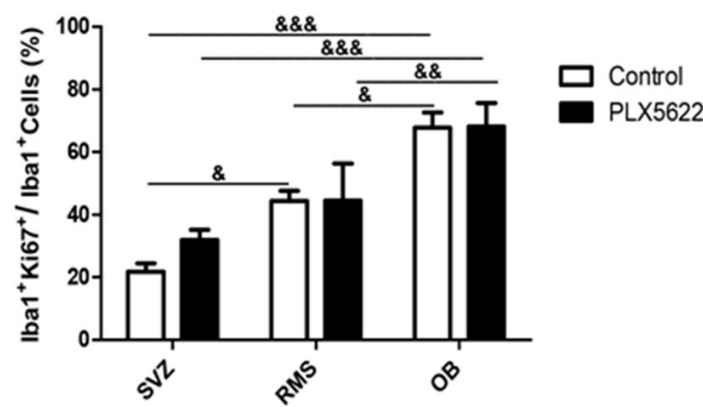

E

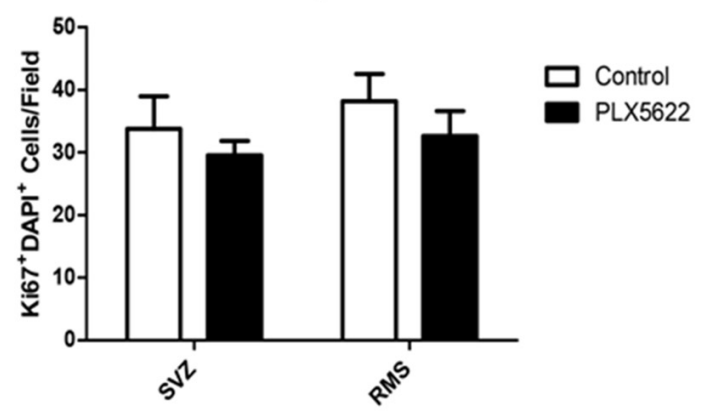

FIGURE 5 | Treatment with PLX5622 has no effect on microglia proliferation. Representative images of the SVZ, RMS, and OB from 14d of PLX5622 treatment (A), with the dashed line separating the SVZ from the lateral ventricle. DAPI in blue, Iba1 in green, Ki67 in gray. Quantification of microglia ablation shown in (B, $\left.F_{(2,15)}=55.46, p<0.0001\right)$. Ki67+l Iba1+ cells were counted $\left(\mathbf{C}, F_{(2,15)}=64.52, p<0.0001\right)$ and normalized $\left(\mathbf{D}, F_{(2,15)}=12.77, p=0.0006\right)$. Similar to the BrdU data, the OB contained more Ki67 ${ }^{+}$microglia than the RMS and SVZ in both control and PLX5622 treated mice. Total Ki67 ${ }^{+}$cells from the SVZ and RMS were quantified in $\left(\mathbf{E}, F_{(2,15)}=0.11, p=0.74\right)$. \& = comparison between regions within treatment, ${ }^{\&}=p<0.5 ; \& \&=p<0.01 ; \& \&=p<0.001$. $n=6$ for all groups.

spine density were observed (Reshef et al., 2017). Another group reported no changes in caspase-3 gene expression using RT-PCR data after 3 weeks of PLX5622 treatment (Walter and Crews, 2017). Similarly, we found no change in the number of apoptotic cells in the SVZ or RMS after 14d of treatment (Supplementary Figure S4). The differences among these reports remain unclear. One possible explanation may be that there are increased numbers of other cell types (GFAP+ or oligo precursors) that are expressing BrdU in the OB; if so, further characterization of the $\mathrm{OB}$ after the various microglial ablation methods is warranted. Regardless, these reports suggest that at brain homeostasis, microglia in the $\mathrm{OB}$ may have an important active role in regulating neural circuits. In support of this, microglia in the OB have a higher turnover rate than other regions of the brain, including the cortex and the hippocampus (Tay et al., 2017). Interestingly, 
our data show that PLX5622 treatment was not as effective in the $\mathrm{OB}$ compared to the SVZ (Figure 1D). Accordingly, the PLX5622 treated OB had no differences in $\mathrm{BrdU}^{+}$or $\mathrm{Ki}^{+} 7^{+}$microglia compared to control (Figures 4B, 5D). It is possible that, due to their high activity at homeostasis in the $\mathrm{OB}$, microglia are stimulated via a route different than CSF1R stimulation. However, another likely explanation is that the turnover rate of $\mathrm{OB}$ microglia is high enough to partially compensate for the PLX5622 CSF1R antagonist. With PLX5622, our data suggest that CSF1R inhibition does not affect the rate of remaining microglia proliferation/turnover (Figures 4B, 5D). The remaining microglia still express CSF1R (Supplementary Figures S6A,B), and would therefore be susceptible to PLX5622. A recent discovery is that microglia repopulation after PLX5622 treatment ensues from the remaining resident populations of microglia after ablation, and not from a progenitor or peripheral source (Huang et al., 2018), also suggesting that the remaining microglia are still capable to proliferate. A different CSF1R inhibitor, the tyrosine kinase inhibitor GW2580, has been reported to decrease CSF1R+ cell proliferation. However, GW2580 does not cause microglia ablation (Olmos-Alonso et al., 2016).

In conclusion, the results presented here show that PLX5622induced brain-wide ablation of microglia for up to 14 days does not significantly modify SVZ-OB adult neurogenesis. CSF1R inhibition via PLX5622 did not change microglia proliferation rates in the SVZ, RMS, or OB. The microglial population in the $\mathrm{OB}$ is unique compared to the SVZ and RMS in its ability to compensate for the CSF1R inhibition. These findings

\section{REFERENCES}

Aarum, J., Sandberg, K., Haeberlein, S. L., and Persson, M. A. (2003). Migration and differentiation of neural precursor cells can be directed by microglia. Proc. Natl. Acad. Sci. U S A 100, 15983-15988. doi: 10.1073/pnas.2237050100

Acharya, M. M., Green, K. N., Allen, B. D., Najafi, A. R., Syage, A., Minasyan, H., et al. (2016). Elimination of microglia improves cognitive function following cranial irradiation. Sci. Rep. 6:31545. doi: 10.1038/srep31545

Arvidsson, A., Collin, T., Kirik, D., Kokaia, Z., and Lindvall, O. (2002). Neuronal replacement from endogenous precursors in the adult brain after stroke. Nat. Med. 8, 963-970. doi: 10.1038/nm747

Beckmann, N., Giorgetti, E., Neuhaus, A., Zurbruegg, S., Accart, N., Smith, P., et al. (2018). Brain region-specific enhancement of remyelination and prevention of demyelination by the CSF1R kinase inhibitor BLZ945. Acta Neuropathol. Commun. 6:9. doi: 10.1186/s40478-018-0510-8

Bond, A. M., Ming, G. L., and Song, H. (2015). Adult mammalian neural stem cells and neurogenesis: five decades later. Cell Stem Cell 17, 385-395. doi: 10.1016/j. stem.2015.09.003

Butovsky, O., Ziv, Y., Schwartz, A., Landa, G., Talpalar, A. E., Pluchino, S., et al. (2006). Microglia activated by IL- 4 or IFN- $\gamma$ differentially induce neurogenesis and oligodendrogenesis from adult stem/progenitor cells. Mol. Cell. Neurosci. 31, 149-160. doi: 10.1016/j.mcn.2005.10.006

Chalmers, S. A., Wen, J., Shum, J., Doerner, J., Herlitz, L., and Putterman, C. (2017). CSF-1R inhibition attenuates renal and neuropsychiatric disease in murine lupus. Clin. Immunol. 185, 100-108. doi: 10.1016/j.clim.2016.08.019

Dagher, N. N., Najafi, A. R., Kayala, K. M., Elmore, M. R., White, T. E., Medeiros, R., et al. (2015). Colony-stimulating factor 1 receptor inhibition prevents microglial plaque association and improves cognition in $3 \times \mathrm{Tg}-\mathrm{AD}$ mice. J. Neuroinflammation 12:139. doi: 10.1186/s12974-015-0366-9

Ekdahl, C. T., Claasen, J. H., Bonde, S., Kokaia, Z., and Lindvall, O. (2003). Inflammation is detrimental for neurogenesis in adult brain. are important for furthering our understanding of microglia in specific regions of the brain, and how responsive local neurogenesis/microglia may be if targeted in cases of brain disease or injury.

\section{DATA AVAILABILITY}

All datasets generated for this study are included in the manuscript and/or the Supplementary Files.

\section{AUTHOR CONTRIBUTIONS}

JK designed and carried out the experiments, collected and analyzed the data, and wrote drafts and edited the manuscript. MW and SG collected and analyzed the data. ST designed the experiments, edited drafts of the manuscript.

\section{FUNDING}

This work was partially supported by an Award from the American Heart Association and the William Randolph Hearst Foundation to JK \#18PRE34030339 and SBMS, National Institutes of Health T32GM127253 to ST.

\section{SUPPLEMENTARY MATERIAL}

The Supplementary Material for this article can be found online at: https://www.frontiersin.org/articles/10.3389/fncel. 2019.00097/full\#supplementary-material

Proc. Natl. Acad. Sci. U S A 100, 13632-13637. doi: 10.1073/pnas.22340 31100

Elmore, M. R., Najafi, A. R., Koike, M. A., Dagher, N. N., Spangenberg, E. E., Rice, R. A., et al. (2014). Colony-stimulating factor 1 receptor signaling is necessary for microglia viability, unmasking a microglia progenitor cell in the adult brain. Neuron 82, 380-397. doi: 10.1016/j.neuron.2014.02.040

Fourgeaud, L., Traves, P. G., Tufail, Y., Leal-Bailey, H., Lew, E. D., Burrola, P. G., et al. (2016). TAM receptors regulate multiple features of microglial physiology. Nature 532, 240-244. doi: 10.1038/nature17630

Gerber, Y. N., Saint-Martin, G. P., Bringuier, C. M., Bartolami, S., Goze-Bac, C., Noristani, H. N., et al. (2018). CSF1R inhibition reduces microglia proliferation, promotes tissue preservation and improves motor recovery after spinal cord injury. Front. Cell. Neurosci. 12:368. doi: 10.3389/fncel.2018.00368

Groh, J., Klein, D., Berve, K., West, B. L., and Martini, R. (2019). Targeting microglia attenuates neuroinflammation-related neural damage in mice carrying human PLP1 mutations. Glia 67, 277-290. doi: 10.1002/glia.23539

Han, J., Harris, R. A., and Zhang, X. M. (2017). An updated assessment of microglia depletion: current concepts and future directions. Mol. Brain 10:25. doi: 10.1186/s13041-017-0307-x

Huang, Y., Xu, Z., Xiong, S., Sun, F., Qin, G., Hu, G., et al. (2018). Repopulated microglia are solely derived from the proliferation of residual microglia after acute depletion. Nat. Neurosci. 21, 530-540. doi: 10.1038/s41593-018-0090-8

Jin, W. N., Shi, S. X., Li, Z., Li, M., Wood, K., Gonzales, R. J., et al. (2017). Depletion of microglia exacerbates postischemic inflammation and brain injury. J. Cereb. Blood Flow Metab. 37, 2224-2236. doi: 10.1177/0271678x17694185

Kokaia, Z., and Lindvall, O. (2003). Neurogenesis after ischaemic brain insults. Curr. Opin. Neurobiol. 13, 127-132. doi: 10.1016/s0959-4388(03)00017-5

Li, Q., and Barres, B. A. (2018). Microglia and macrophages in brain homeostasis and disease. Nat. Rev. Immunol. 18, 225-242. doi: 10.1038/nri.2017.125

Lois, C., García-Verdugo, J. M., and Alvarez-Buylla, A. (1996). Chain migration of neuronal precursors. Science 271, 978-981. doi: 10.1126/science.271.5251.978 
Menn, B., Garcia-Verdugo, J. M., Yaschine, C., Gonzalez-Perez, O., Rowitch, D., and Alvarez-Buylla, A. (2006). Origin of oligodendrocytes in the subventricular zone of the adult brain. J. Neurosci. 26, 7907-7918. doi: 10.1523/JNEUROSCI. 1299-06.2006

Nelson, L. H., and Lenz, K. M. (2017). Microglia depletion in early life programs persistent changes in social, mood-related, and locomotor behavior in male and female rats. Behav. Brain Res. 316, 279-293. doi: 10.1016/j.bbr.2016.09.006

Nimmerjahn, A., Kirchhoff, F., and Helmchen, F. (2005). Resting microglial cells are highly dynamic surveillants of brain parenchyma in vivo. Science 308, 1314-1318. doi: 10.1126/science.1110647

Nissen, J. C., Thompson, K. K., West, B. L., and Tsirka, S. E. (2018). Csf1R inhibition attenuates experimental autoimmune encephalomyelitis and promotes recovery. Exp. Neurol. 307, 24-36. doi: 10.1016/j.expneurol.2018. 05.021

Olmos-Alonso, A., Schetters, S. T., Sri, S., Askew, K., Mancuso, R., VargasCaballero, M., et al. (2016). Pharmacological targeting of CSF1R inhibits microglial proliferation and prevents the progression of Alzheimer's-like pathology. Brain 139, 891-907. doi: 10.1093/brain/awv379

Ortega, F., Gascon, S., Masserdotti, G., Deshpande, A., Simon, C., Fischer, J., et al. (2013). Oligodendrogliogenic and neurogenic adult subependymal zone neural stem cells constitute distinct lineages and exhibit differential responsiveness to Wnt signalling. Nat. Cell Biol. 15, 602-613. doi: 10.1038/ncb2736

Paolicelli, R. C., Bolasco, G., Pagani, F., Maggi, L., Scianni, M., Panzanelli, P., et al. (2011). Synaptic pruning by microglia is necessary for normal brain development. Science 333, 1456-1458. doi: 10.1126/science.1202529

Parent, J. M. (2003). Injury-induced neurogenesis in the adult mammalian brain. Neuroscientist 9, 261-272. doi: 10.1177/1073858403252680

Petreanu, L., and Alvarez-Buylla, A. (2002). Maturation and death of adult-born olfactory bulb granule neurons: role of olfaction. J. Neurosci. 22, 6106-6113. doi: 10.1523/JNEUROSCI.22-14-06106.2002

Reshef, R., Kreisel, T., Beroukhim Kay, D., and Yirmiya, R. (2014). Microglia and their CX3CR1 signaling are involved in hippocampal- but not olfactory bulb-related memory and neurogenesis. Brain Behav. Immun. 41, 239-250. doi: 10.1016/j.bbi.2014.04.009

Reshef, R., Kudryavitskaya, E., Shani-Narkiss, H., Isaacson, B., Rimmerman, N., Mizrahi, A., et al. (2017). The role of microglia and their CX3CR1 signaling in adult neurogenesis in the olfactory bulb. Elife 6:e30809. doi: 10.7554/elife. 30809

Ribeiro Xavier, A. L., Kress, B. T., Goldman, S. A., Lacerda de Menezes, J. R., and Nedergaard, M. (2015). A distinct population of microglia supports adult neurogenesis in the subventricular zone. J. Neurosci. 35, 11848-11861. doi: 10.1523/JNEUROSCI.1217-15.2015

Scott, R. S., McMahon, E. J., Pop, S. M., Reap, E. A., Caricchio, R., Cohen, P. L., et al. (2001). Phagocytosis and clearance of apoptotic cells is mediated by MER. Nature 411, 207-211. doi: 10.1038/35075603

Seitz, S., Clarke, P., and Tyler, K. L. (2018). Pharmacologic depletion of microglia increases viral load in the brain and enhances mortality in murine models of flavivirus-induced encephalitis. J. Virol. 92:e00525-18. doi: 10.1128/jvi. 00525-18
Shigemoto-Mogami, Y., Hoshikawa, K., Goldman, J. E., Sekino, Y., and Sato, K. (2014). Microglia enhance neurogenesis and oligodendrogenesis in the early postnatal subventricular zone. J. Neurosci. 34, 2231-2243. doi: 10.1523/JNEUROSCI.1619-13.2014

Sierra, A., Encinas, J. M., Deudero, J. J., Chancey, J. H., Enikolopov, G., Overstreet-Wadiche, L. S., et al. (2010). Microglia shape adult hippocampal neurogenesis through apoptosis-coupled phagocytosis. Cell Stem Cell 7, 483-495. doi: 10.1016/j.stem.2010.08.014

Sobecki, M., Mrouj, K., Camasses, A., Parisis, N., Nicolas, E., Llères, D., et al. (2016). The cell proliferation antigen Ki-67 organises heterochromatin. Elife 5:e13722. doi: 10.7554/eLife.13722

Szalay, G., Martinecz, B., Lénárt, N., Környei, Z., Orsolits, B., Judák, L., et al. (2016). Microglia protect against brain injury and their selective elimination dysregulates neuronal network activity after stroke. Nat. Commun. 7:11499. doi: 10.1038/ncomms11499

Takahashi, K., Rochford, C. D., and Neumann, H. (2005). Clearance of apoptotic neurons without inflammation by microglial triggering receptor expressed on myeloid cells-2. J. Exp. Med. 201, 647-657. doi: 10.1084/jem.20041611

Tay, T. L., Mai, D., Dautzenberg, J., Fernández-Klett, F., Lin, G., Sagar, et al. (2017). A new fate mapping system reveals context-dependent random or clonal expansion of microglia. Nat. Neurosci. 20, 793-803. doi: 10.1038/nn.4547

Torres, L., Danver, J., Ji, K., Miyauchi, J. T., Chen, D., Anderson, M. E., et al. (2016). Dynamic microglial modulation of spatial learning and social behavior. Brain Behav. Immun. 55, 6-16. doi: 10.1016/j.bbi.2015.09.001

Unger, M. S., Schernthaner, P., Marschallinger, J., Mrowetz, H., and Aigner, L. (2018). Microglia prevent peripheral immune cell invasion and promote an anti-inflammatory environment in the brain of APP-PS1 transgenic mice. J. Neuroinflammation 15:274. doi: 10.1186/s12974-018-1304-4

Wake, H., Moorhouse, A. J., Miyamoto, A., and Nabekura, J. (2013). Microglia: actively surveying and shaping neuronal circuit structure and function. Trends Neurosci. 36, 209-217. doi: 10.1016/j.tins.2012.11.007

Walter, T. J., and Crews, F. T. (2017). Microglial depletion alters the brain neuroimmune response to acute binge ethanol withdrawal. J. Neuroinflammation 14:86. doi: 10.1186/s12974-017-0856-Z

Yao, Y., Echeverry, S., Shi, X. Q., Yang, M., Yang, Q. Z., Wang, G. Y., et al. (2016). Dynamics of spinal microglia repopulation following an acute depletion. Sci. Rep. 6:22839. doi: 10.1038/srep22839

Conflict of Interest Statement: The authors declare that the research was conducted in the absence of any commercial or financial relationships that could be construed as a potential conflict of interest.

Copyright (c) 2019 Kyle, Wu, Gourzi and Tsirka. This is an open-access article distributed under the terms of the Creative Commons Attribution License (CC BY). The use, distribution or reproduction in other forums is permitted, provided the original author(s) and the copyright owner(s) are credited and that the original publication in this journal is cited, in accordance with accepted academic practice. No use, distribution or reproduction is permitted which does not comply with these terms. 